\title{
PNPLA3 I148M gene variant and Chronic Kidney Disease in Type 2 \\ Diabetic Patients With NAFLD: Clinical and Experimental Findings
}

Short title: PNPLA3 and decreased kidney function

Alessandro Mantovani, $\mathrm{MD}^{1}$, Alice Taliento, $\mathrm{BS}^{2}$, Chiara Zusi, $\mathrm{PhD}^{1,3}$, Guido Baselli, $\mathrm{PhD}^{4}$, Daniele Prati, $\mathrm{MD}^{4}$, Simona Granata, $\mathrm{PhD}^{5}$, Gianluigi Zaza, $\mathrm{MD}^{5}$, Antonio Colecchia, $\mathrm{MD}^{6}$, Claudio Maffeis, $\mathrm{MD}^{3}$, Christopher D. Byrne, MB BCh, $\mathrm{PhD}^{7,8}$, Luca Valenti, MD²,4§, Giovanni Targher, $\mathrm{MD}^{1 \S}$

${ }^{1}$ Division of Endocrinology, Diabetes and Metabolism, Department of Medicine, University Hospital of Verona, Verona, Italy

${ }^{2}$ Translational Medicine, Department of Transfusion Medicine and Hematology, Fondazione IRCCS Ca' Granda Ospedale Maggiore Policlinico, Milan, Italy

${ }^{3}$ Pediatric Diabetes and Metabolic Disorders Unit, Department of Surgical Sciences, Dentistry, Pediatrics and Gynaecology, University Hospital of Verona, Verona, Italy

${ }^{4}$ Department of Pathophysiology and Transplantation, University of Milan, Milan, Italy

${ }^{5}$ Renal Unit, Department of Medicine, University and Azienda Ospedaliera Universitaria Integrata of Verona, Verona, Italy

${ }^{6}$ Gastroenterology Unit, Azienda Ospedaliera Universitaria Integrata of Verona, Verona, Italy ${ }^{7}$ Nutrition and Metabolism, Faculty of Medicine, University of Southampton, Southampton, UK

${ }^{8}$ Southampton National Institute for Health Research Biomedical Research Centre, University Hospital Southampton, Southampton General Hospital, Southampton, UK

$\S$ These authors contributed equally to this work

Word count: unstructured abstract 250; text 4,812 (excluding title page, abstract, references, figure legends and tables); n. 3 Tables; n. 2 Figures; $n$. 1 supplementary Table

Key-words: adiponutrin; kidney dysfunction; NAFLD; renal podocytes

\author{
Address of correspondence: \\ Prof. Giovanni Targher, MD \\ Section of Endocrinology, Diabetes and Metabolism \\ University and Azienda Ospedaliera Universitaria Integrata \\ Piazzale Stefani, 1 \\ 37126 Verona, Italy \\ Phone: $+39 / 045-8123748$ \\ E-mail: giovanni.targher@univr.it
}




\section{ABBREVIATION LIST}

$A C R$, albumin-to-creatinine ratio

$A L T$, alanine aminotransferase

AST, aspartate aminotransferase

BMI, body mass index

CKD-EPI, chronic kidney disease Epidemiology Collaboration

CKD, chronic kidney disease

e-GFR, estimated glomerular filtration rate

LSM, liver stiffness measurement

MDRD, Modification of Diet in Renal Disease

mRNA, messenger RNA

NAFLD, nonalcoholic fatty liver disease

PNPLA3, patatin-like phospholipase domain-containing protein 3

HOMA-IR, homeostasis model assessment-insulin resistance

hs-CRP, high sensitivity C-reactive protein

GGT, gamma-glutamyltransferase

T2DM, type 2 diabetes mellitus

Disclosure Statement: The Authors have no potential conflicts of interest to disclose.

Funding: GT is supported in part by grants from the University School of Medicine of Verona, Verona, Italy. CDB is supported in part by grants from the Southampton National Institute for Health Research Biomedical Research Centre. LV was supported by MyFirst Grant AIRC $n$. 16888, Ricerca Finalizzata Ministero della Salute RF-2016-02364358, Ricerca corrente Fondazione IRCCS Ca' Granda Ospedale Maggiore Policlinico, the European Union (EU) Programme Horizon 2020 (under grant agreement No. 777377) for the project LITMUS - "Liver Investigation: Testing Marker Utility in Steatohepatitis".

Authors' Contributions: GT and LV conceived and designed the study. AM, AT, CZ, GB and DP researched data and reviewed/edited the manuscript. SG, GZ, AC, CM and CDB contributed to discussion and reviewed/edited the manuscript. AM, AT, LV and GT analyzed the data. AM and GT wrote the manuscript. GT and LV are the guarantors of this work and, as such, had full access to all the data of the study and take responsibility for the integrity and accuracy of data. All authors approved the final version of the manuscript. 


\section{ABSTRACT}

Background \& Aims: Emerging evidence suggests an association between patatin-like phospholipase domain-containing protein-3 (PNPLA3) rs738409 (I148M protein variant), and risk of chronic kidney disease (CKD), but the mechanisms underpinning this association are poorly understood.

Methods: We studied 157 patients with type 2 diabetes (T2DM) who underwent ultrasonography and vibration-controlled transient elastography for diagnosing nonalcoholic fatty liver disease (NAFLD). CKD was defined as estimated glomerular filtration rate (e-GFR) $<60 \mathrm{ml} / \mathrm{min} / 1.73 \mathrm{~m}^{2}$ and/or abnormal albuminuria. We surveyed PNPLA3 mRNA expression in human tissues, using the liver as a positive control, and also measured PNPLA3 mRNA and protein expression levels in human cell lines represented in the kidney and the liver.

Results: 112 patients had NAFLD and 43 had CKD. Patients homozygous for the I148M variant $(n=11)$ had lower e-GFR levels $\left(60.6 \pm 11.7\right.$ vs. $77.8 \pm 15.9$ vs. $83.5 \pm 16.5 \mathrm{ml} / \mathrm{min} / 1.73 \mathrm{~m}^{2}$, $p=0.0001)$ and higher prevalence of CKD (63.6\% vs. $24.2 \%$ vs. $25.0 \%, p=0.028)$, compared to those with I/M ( $\mathrm{n=66)}$ and I/I ( $=80)$ PNPLA3 genotype. The association of I148M homozygosity with lower e-GFR levels $(p<0.0001)$ and higher risk of CKD (adjusted-odds ratio 6.65; 95\% Cl 1.65-26.8, $p=0.008$ ) was independent of liver disease severity (as detected by liver stiffness $\geq 7 \mathrm{kPa}$ ) and other risk factors. PNPLA3 mRNA expression was greatest in liver and renal cortex, and podocytes showed high PNPLA3 mRNA and protein levels, comparable to that of hepatocytes and hepatic stellate cells, respectively.

Conclusions: The PNPLA3 I148M variant was associated with CKD, independently of common renal risk factors and severity of NAFLD. PNPLA3 expression levels were particularly high in renal podocytes. 


\section{INTRODUCTION}

Non-alcoholic fatty liver disease (NAFLD) and chronic kidney disease (CKD) are two worldwide public health problems affecting up to nearly $25 \%$ (NAFLD) and up to nearly $15 \%$ (CKD) of adults in Western countries (1-4). Both diseases are progressive chronic conditions that represent a spectrum of diseases extending from relatively mild disease, with only modest changes in function, to severe debilitating disease with end-stage organ damage, necessitating either dialysis or organ transplantation in order to sustain life. The worldwide rise in the number of individuals with CKD and consequent kidney failure necessitating renal replacement therapy is threatening to reach epidemic proportions over the next decade $(3,4)$. Importantly, the presence of CKD not only increases the risk of developing end-stage renal disease but also the risk of developing cardiovascular disease and other comorbidities (5). For this reason, the early identification of acquired and genetic risk factors for CKD is crucial in clinical practice.

Several susceptibility gene variants predisposing to NAFLD have been identified in different populations (6-8). Among the genetic factors that influence the development and progression of NAFLD, the rs738409 C>G single nucleotide polymorphism in the patatin-like phospholipase domain-containing protein-3 (PNPLA3) gene, encoding for the p.lle148Met change (I148M), is the single genetic variant accounting for the largest proportion of NAFLD risk in the population (6-8).

A large body of evidence supports the existence of an association between the presence and severity of NAFLD and increased prevalence and incidence of CKD, independent of obesity, type 2 diabetes mellitus (T2DM), hypertension and other common cardio-metabolic risk 
factors (9-16). Emerging evidence suggests that the PNPLA3 I148M variant is also associated with decreasing levels of estimated glomerular filtration rate (e-GFR) or presence of CKD, irrespective of common renal risk factors and presence or severity of NAFLD in both adults and children (17-23).

Presently, however, there are very few studies that have examined the association between the PNPLA3 I148M variant and decreased kidney function among patients with T2DM (19). This is important because patients with T2DM represent a group of individuals, who are at high risk of developing both CKD and the more severe forms of NAFLD (that also includes hepatocellular carcinoma) (1-3). In addition, the putative mechanisms underpinning the association between the PNPLA3 I148M variant and risk of kidney damage are poorly understood. In particular, it is not known whether the increased predisposition to kidney damage in carriers of the I148M variant is mediated through NAFLD or by other pleiotropic effects, including a possible direct role in the kidney. Emerging evidence suggests that renal podocytes accumulate lipid droplets, and this process is associated with NLRP3 inflammasome activation and is involved in the pathophysiology of diabetic nephropathy (24).

Thus, the major aims of our clinical and experimental study were firstly to investigate the association between the PNPLA3 I148M variant and risk of CKD in an outpatient sample of T2DM individuals without known liver diseases. Having demonstrated an association between CKD and PNPLA3 I148M, our second aim was to examine whether PNPLA3 gene and protein expression are detectable in the kidney. Specifically, for this second aim, we assessed PNPLA3 mRNA and protein levels in various human tissues, performing experiments in human cell lines that included both kidney and liver cell populations. 


\section{MATERIALS AND METHODS}

\section{Patients}

We enrolled 157 Caucasian patients with T2DM, who consecutively attended our secondary care diabetes service over a 6-month period, and who accepted to undergo liver ultrasonography and vibration-controlled transient elastography for diagnosing and staging NAFLD. Of these patients, 77 women with T2DM have been included in a previously published study in which we investigated relationships between PNPLA3 polymorphism and CKD in postmenopausal women (19). We excluded all patients with: (a) history of significant alcohol consumption and other known causes of chronic liver diseases (e.g., virus, drugs, autoimmunity and hemochromatosis); (b) cirrhosis of any aetiology, active cancer and endstage kidney disease (defined as e-GFR $<15 \mathrm{ml} / \mathrm{min} / 1.73 \mathrm{~m}^{2}$ or dialysis); (c) chronic use of potentially hepatotoxic drugs, including steroids, tamoxifen, amiodarone, methotrexate or treatment with hormone replacement therapy (only for women); and (d) treatment with insulin. We excluded patients on insulin treatment (i.e., an established risk factor for osteoporosis and bone fractures), because this study was primarily designed for exploring the association between NAFLD and bone turnover biomarkers (25). According to the technical limitations of the vibration-controlled transient elastography, patients with congestive heart failure or free abdominal fluid were also excluded from the study.

The study protocol was approved by the local Ethics Committee of the Hospital Trust of Verona. All patients gave their written informed consent for participation in this research.

Clinical and laboratory data 
Body mass index (BMI) was measured as kilograms divided by the square of height in meters. Waist circumference was measured at the midpoint between the lowest rib and the iliac crest. Patients were considered to have hypertension if their blood pressure was $\geq 140 / 90 \mathrm{mmHg}$ or if they were taking any anti-hypertensive agents.

Venous blood samples were collected in the morning after an overnight fast. Complete blood count, liver enzymes (aspartate aminotransferase [AST], alanine aminotransferase [ALT], gamma-glutamyltransferase [GGT]), creatinine (measured using a Jaffé rate blanked and compensated assay), high-sensitivity $\mathrm{C}$ reactive protein (hs-CRP) and other biochemical parameters were measured by standard laboratory procedures at the Central Laboratory of our hospital, using the relative reference techniques on Roche Cobas ${ }^{\circledR} 8000$ (Roche Diagnostics, Basel, Switzerland). Low-density lipoprotein (LDL)-cholesterol was calculated using the Friedewald's equation. Hemoglobin A1c (HbA1c) was measured using the highperformance liquid chromatography analyzer Tosoh-G7 (Tosoh Bioscience Inc., Tokyo, Japan). Fasting insulin was measured using a chemiluminescent immunoassay (LIAISON, DiaSorin, Saluggia, Italy). Homeostasis model assessment (HOMA-IR) score was used for estimating insulin resistance.

e-GFR was estimated using both the CKD-Epidemiology Collaboration (CKD-EPI) equation (26) and the four-variable Modification of Diet in Renal Disease (MDRD) study equation (27). Albuminuria was measured with an immuno-nephelometric assay (Beckman-Coulter IMMAGE; Beckman-Coulter Instruments, Fullerton, CA, USA) on a morning spot urine sample and expressed as the albumin-to-creatinine ratio (ACR); abnormal albuminuria was defined as urinary $A C R \geq 30 \mathrm{mg} / \mathrm{g}$ creatinine. Timed or 24 -h urine collections are more burdensome and 
add little to prediction or accuracy of albuminuria assessment compared with spot urinary ACR (28). In this study, CKD was defined as the presence of e-GFR $<60 \mathrm{ml} / \mathrm{min} / 1.73 \mathrm{~m}^{2}$ and/or urinary ACR $\geq 30 \mathrm{mg} / \mathrm{g}$ creatinine (28).

Presence of ischemic heart disease was defined as a documented history of myocardial infarction, angina or coronary revascularization procedures. Presence of diabetic retinopathy (diagnosed with fundoscopy after pupillary dilation) was also recorded in all patients.

\section{Ultrasonography and liver stiffness measurement}

A trained physician, who was blinded to participants' clinical and biochemical details, performed both ultrasonography and liver stiffness measurement (LSM) in all patients after at least 8 hours of fasting.

Hepatic steatosis was diagnosed by ultrasonography (using an Esaote MyLab 70 ultrasound with a $4 \mathrm{MHz}$ probe) according to specific ultrasonographic characteristics, such as diffuse hyperechogenicity of the liver relative to kidneys, ultrasonographic beam attenuation, and poor visualization of intra-hepatic vessel borders and diaphragm. The ultrasonographic severity of hepatic steatosis was expressed with a semi-quantitative scale (i.e., mild, moderate or severe) (19).

LSMs were performed using vibration-controlled transient elastography (FibroScan ${ }^{\circledR}$ Echosens, Paris, France) with the $\mathrm{M}$ probe. Further details of the technical examination procedures have been described elsewhere $(29,30)$. In brief, each LSM measurement was considered adequate when including at least 10 valid measurements for each patient, with a success rate $>60 \%$ and 
variability of measurements $(I Q R / M)<30 \%$ of the median. The presence of significant liver fibrosis was defined by the presence of $L S M \geq 7 \mathrm{kPa}$ (that corresponds to the Kleiner fibrosis stage $F \geq 2$ on liver histology) (30). Unfortunately, we did not have the FibroScan ${ }^{\circledR} \mathrm{XL}$ probe. Although the FibroScan ${ }^{\circledast} \mathrm{XL}$ probe reduces transient elastography failure and facilitates reliable LSM in severely obese patients, both the $\mathrm{M}$ and $\mathrm{XL}$ probes have comparable accuracy for diagnosing significant liver fibrosis (31). The applicability and accuracy of the FibroScan ${ }^{\circledR} \mathrm{M}$ probe to identify significant fibrosis is excellent in patients with overweight or grade 1 obesity $\left(\mathrm{BMI} \leq 35 \mathrm{~kg} / \mathrm{m}^{2}\right)(32)$. In this study only $11(7 \%)$ patients had a $\mathrm{BMI}>35 \mathrm{~kg} / \mathrm{m}^{2}$. Our FibroScan ${ }^{\circledR}$ device was not equipped with the controlled attenuation parameter (CAP) for measuring hepatic steatosis. However, Carvalhana et al. reported a good correlation (Spearman's $\rho=0.75$, $p<0.0001$ ) between ultrasound and CAP when comparing the diagnostic accuracy of these two techniques for detecting hepatic steatosis (33).

\section{Genetic analysis}

In all participants genomic DNA was extracted from peripheral blood leukocytes using the QIAamp DNA Blood Mini Kit (Qiagen, Germany). Genotyping of rs738409 in patatin-like phospholipase domain-containing protein-3 (PNPLA3) gene (I148M variant) was carried out by a predesigned TaqMan probe (Applied Biosystem, California, USA), according to manufacturer's protocol. Single nucleotide polymorphism (SNP) genotyping was performed using 7900 HT Real-Time PCR (Applied Biosystem, California, USA). The call rate exceeded 99\% for both SNPs and genotypes' distributions were in Hardy-Weinberg equilibrium. The minor allele frequency of PNPLA3 rs738409 was $28 \%$. 
PNPLA3 gene expression data were retrieved from the BioGPS database across 262 normal human organs and tissues, where they were evaluated by the U133plus2 Affymetrix microarray (http://biogps.org/\#goto=genereport\&id=80339). The values are represented as Zscores that were produced by the Gene Expression Barcode method (34). According to this standardization method, a Z-score $>5$ indicates that an annotated gene is expressed at significant level in a specific tissue.

\section{Cell cultures}

We selected human cell lines as models of cell types represented both in the liver (as a positive control) and in the kidney to assess PNPLA3 expression. HepG2 (hepatoma cells), LX2 (hepatic stellate cells), and conditionally immortalized human podocyte cell lines $(35,36)$ were cultured in DMEM (Life Technologies, USA) supplemented with 10\% FBS (Life Technologies, USA), 1\% L-glutamine (Life Technologies, USA) and 1\% penicillin streptomycin (Life Technologies, USA). HK2 (kidney tubular cells) were cultured in DMEM F12 (Life Technologies, USA) supplemented with 10\% FBS (Life Technologies, USA), 1\% L-Glutamine (Life Technologies, USA) and 1\% penicillin streptomycin (Life Technologies, USA). Human umbilical vein endothelial cells (HUVEC), as a model for endothelial cells expressed in renal glomeruli, were cultured in Endothelial Cell Growth Medium-2 (EGM2) (Lonza, Switzerland).

\section{Gene expression evaluation}

Total RNA was extracted from cells using the Trizol reagent (Life Technologies, USA) and messenger RNA (mRNA) was retro-transcribed by VILO IV Superscript Kit with ezDNAse (Life Technologies, USA). The mRNA expression levels of PNPLA3 were determined using TaqMan probes (Life Technologies, USA) according to the manufacturer's protocol. Experiments were 
assayed in triplicate and repeated twice. PNPLA3 gene expression was normalized for betaactin, as the reference gene.

\section{Immunoblotting}

Cell lines were lysed and proteins were separated by electrophoresis and then transferred to nitrocellulose membranes for Western blotting. The following antibodies were used: antiPNPLA3 (ab81874, Abcam, UK) and anti- $\beta$ actin (Cell Signaling, USA). Protein expression levels were quantified using ImageJ Software (https://imagej.nih.gov/ij/download.html).

\section{Statistical analysis}

Data are expressed as means $\pm S D$, medians (interquartile ranges [IQRs]) or percentages. The Fisher's exact test was used for categorical variables, the one-way analysis of variance (ANOVA) was used for normally distributed continuous variables and the Kruskal-Wallis test was used for non-normally distributed variables (i.e., diabetes duration, fasting insulin, HOMA-IR score, hs-CRP and GGT levels), to investigate the differences in clinical and biochemical characteristics among patients with T2DM, stratified by the PNPLA3 rs738409 (I148M). The association between the PNPLA3 I148M variant and levels of e-GFR (included as a continuous measure, i.e., for each $1 \mathrm{ml} / \mathrm{min} / 1.73 \mathrm{~m}^{2}$ decrement) was tested using both an unadjusted linear regression model and a multivariable linear regression model adjusted for diabetes duration, $\mathrm{HbA1c}$, hypertension (i.e., blood pressure $\geq 140 / 90 \mathrm{mmHg}$ or use of any anti-hypertensive drugs), presence of significant fibrosis (i.e., LSM $\geq 7 \mathrm{kPa}$ on FibroScan ${ }^{\circledR}$ ) and abnormal albuminuria. We also tested the association between the PNPLA3 I148M variant and the risk of having CKD (included as a dichotomous variable, i.e., defined as e-GFR CKD-EPI $_{\text {- }} 60 \mathrm{~mL} / \mathrm{min} / 1.73 \mathrm{~m}^{2}$ and/or abnormal albuminuria) by using an unadjusted 
logistic regression model and a multivariable logistic regression model that included the same list of the aforementioned covariates (except for abnormal albuminuria that was included in the CKD definition). The impact of the PNPLA3 I148M variant on levels of e-GFR and risk of CKD was assessed using additive genetic models. Covariates included in the above-mentioned multivariable regression models were selected based on their significance in univariable analyses, or based on their biological plausibility as potential confounding factors. However, in these models we did not include sex and age among the covariates, as these two variables were included in the equation used for estimating e-GFR (and, consequently, also in the CKD definition).

With regards to the experimental part of our study, HepG2 hepatocytes were used as the reference standard to report relative expression. The unpaired Student's $t$-test was used to test differences in PNPLA3 mRNA and protein levels.

All statistical tests were two-sided, and $p$-values $<0.05$ (two-tailed) were considered to be statistically significant. Statistical analyses were performed using STATA software, version 14.2 (STATA, College Station, TX).

\section{RESULTS}

Association between PNPLA3 I148M variant, reduced e-GFR levels and CKD

Overall, 157 Caucasian patients with T2DM (mean age 69 \pm 9 years; $51 \%$ men; mean BMI $28.4 \pm 5 \mathrm{~kg} / \mathrm{m}^{2}$; mean $\mathrm{HbA} 1 \mathrm{c} 6.9 \pm 0.7 \%$ ) without known liver diseases were included in the study. 43 (27.4\%) patients had CKD, $112(71.3 \%)$ had hepatic steatosis on ultrasonography and 
30 (19.1\%) had LSM $\geq 7 \mathrm{kPa}$ on vibration-controlled transient elastography. In addition, 11 (7\%) of these patients had M/M (i.e., homozygous carriers of the I148M variant), 66 (42.1\%) had I/M (heterozygous carriers of the I148M variant), and 80 (50.9\%) had I/I PNPLA3 rs738409 genotype (homozygous carriers of the wild-type 148 l allele), respectively.

The clinical and biochemical characteristics of patients stratified by PNPLA3 I148M variant are listed in Table 1. Patients homozygous for the I148M variant had significantly higher serum levels of triglycerides, GGT and creatinine than those who were not. In addition, among patients homozygous for the I148M variant there was a higher proportion of individuals with e-GFR CKD-EPI $<60 \mathrm{ml} / \mathrm{min} / 1.73 \mathrm{~m}^{2}$, as well as a higher proportion of individuals with CKD (defined as e-GFR CKD-EPI $_{60} \mathrm{ml} / \mathrm{min} / 1.73 \mathrm{~m}^{2}$ and/or abnormal albuminuria).

Age, sex, adiposity measures (including patients with $\mathrm{BMI}>35 \mathrm{~kg} / \mathrm{m}^{2}$ ), duration of diabetes, smoking, blood pressure, white blood cell count, platelet count, hemoglobin, serum transaminases, lipids (except for triglycerides), fasting glucose, insulin, HbA1c, HOMA-IR score, hs-CRP, and proportion of abnormal albuminuria, hypertension, ischemic heart disease and diabetic retinopathy, as well as use of anti-hyperglycemic agents, anti-hypertensive drugs, anti-platelets or statins did not significantly differ among the three groups of patients. In addition, the proportion of hepatic steatosis on ultrasonography and significant fibrosis on FibroScan ${ }^{\circledR}$ was also comparable among the three patient groups.

Notably, as shown in Fig. 1, the values of both e-GFR CKD-EPI $_{\text {(panel A) and e-GFR }}$ MDRD (panel B) decreased significantly across the PNPLA3 genotype, with the lowest e-GFR values among patients homozygous for the I148M variant. 
As reported in Table 2, the risk allele of rs738409, encoding for the I148M protein variant, was significantly associated with lower e-GFR model ( $\beta$ coefficient $-22.9,95 \% \mathrm{Cl}-33.5$ to $-12.3, p<0.0001$ ) and in the adjusted regression model adjusting for diabetes duration, $\mathrm{HbA1c}$, hypertension, abnormal albuminuria, and presence of significant liver fibrosis ( $\beta$ coefficient $-23.2,95 \% \mathrm{Cl}-33.2$ to $-13.2, p<0.0001$ ). The additive effect of each $148 \mathrm{M}$ allele corresponded to a decrement of nearly $9 \mathrm{ml} / \mathrm{min} / 1.73 \mathrm{~m}^{2}$

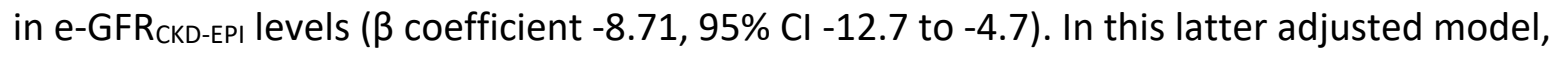
other variables that were independently associated with lower e-GFR diabetes duration ( $\beta$ coefficient $-0.47,95 \% \mathrm{Cl}-0.76$ to $-0.19, p=0.001$ ) and abnormal albuminuria ( $\beta$ coefficient $-6.87,95 \% \mathrm{Cl}-13.2$ to $-0.51, p=0.035$ ).

When we additionally adjusted the above-mentioned regression models for use of statins and glucagon-like peptide-1 receptor agonists (GLP-1RAs) or sodium-glucose cotransporter 2 (SGLT-2) inhibitors (i.e., two classes of antihyperglycemic drugs that have been reported to improve renal outcomes in large randomized controlled trials in T2DM), the risk allele of rs738409 remained significantly associated with lower e-GFR CKD-EPI levels (adjusted $\beta$ coefficient $-23.3,95 \% \mathrm{Cl}-33.4$ to $-13.1, p<0.0001)$. Almost identical results were also observed when we used the MDRD study equation, instead of the CKD-EPI equation, for estimating eGFR (data not shown).

Table 3 provides the association between the PNPLA3 I148M variant and risk of having CKD, and the effect of adjustment for established renal risk factors and potential confounders. In univariable regression analysis (unadjusted model), the homozygosity for the I148M variant 
was associated with an approximate five-fold increased risk of having CKD (unadjusted-odds ratio $5.27,95 \% \mathrm{Cl} 1.39-19.8, p=0.014)$. Notably, adjustment for duration of diabetes, HbA1c, hypertension, and presence of significant liver fibrosis did not alter these results.

\section{PNPLA3 is highly expressed in renal podocytes}

To assess whether the increased susceptibility to kidney damage in carriers of the risk allele (G) of rs738409 could be due to a direct effect of the PNPLA3 I148M variant on the kidney, we have also assessed PNPLA3 mRNA expression in human cells from different tissues (see Fig. 2A for the liver and kidney expression and Supplementary Table $\mathbf{1}$ for the whole set of tissues available). Notably, we found that the liver and the kidney were the two organs that expressed highest PNPLA3 mRNA levels (Fig. 2A). Additionally, in the kidney the PNPLA3 mRNA expression was significantly higher in the cortex than in the medulla.

We next examined the relative expression levels of PNPLA3 in human hepatic and renal cells. In particular, we tested hepatocytes (HepG2), hepatic stellate cells (LX2) and, for the kidney, SV40-immortalized podocytes and HK2 tubular cells. As the alteration of the endothelial barrier in the glomeruli has a key role in the pathogenesis of diabetic nephropathy, we also quantified PNPLA3 expression in HUVEC cells. These data showed that PNPLA3 mRNA levels were only slightly lower in renal podocytes than in HepG2 cells, which expressed PNPLA3 most, but mRNA levels were higher in podocytes than in LX2, HK2 and HUVEC cells (Fig. 2B). The high level of PNPLA3 mRNA expression variant in podocytes was not accounted for by carriage of the PNPLA3 I148M. Indeed, whilst HepG2 and LX2 cells were homozygous for the mutant I148M allele, renal podocytes and HK2 cells were homozygous for the wild-type 148I allele (Fig. 2B). 
Finally, we quantified PNPLA3 protein levels in the different cell lines. Remarkably, renal podocytes showed higher levels of PNPLA3 protein compared to HepG2, HK2 and HUVEC cells, with levels that were comparable to those of LX2 cells (Fig. 2C and 2D).

\section{DISCUSSION}

The main and novel findings of our clinical and experimental study were firstly, the carriage of the PNPLA3 rs738409 polymorphism, encoding for the I148M protein variant (i.e., the major genetic variant associated with susceptibility to NAFLD development and progression), was closely associated with decreasing e-GFR levels and an increased prevalence of CKD in patients with T2DM. Patients were not know to have liver disease and these associations were independent of duration of diabetes, glycaemic control, hypertension and presence of significant fibrosis on FibroScan ${ }^{\circledR}$. Secondly, our data show that PNPLA3 mRNA levels were greatest in the liver and in the kidney (in particular in the cortical region), when compared to those in other organ tissues. Thirdly, among the major cellular types of the kidney, podocytes were characterized by higher PNPLA3 mRNA and protein expression levels than tubular cells and other renal cell types. Furthermore, these expression levels were comparable to those of cells, namely hepatocytes and hepatic stellate cells, which mediate the impact of the I148M variant on liver disease.

In this study, the prevalence of hepatic steatosis and raised liver stiffness was not different across PNPLA3 I148M genotypes. This finding seems to be at variance with previous studies 
showing a significant association between PNPLA3 I148M gene variant and risk of NAFLD progression, especially in cohorts of patients with biopsy-confirmed NAFLD $(1,2,6,37)$. In our patients with T2DM who were not selected for the presence of liver diseases, as expected we found a relatively low frequency of individuals with homozygosity for the $148 \mathrm{M}$ variant (i.e., only $7 \%$ of these T2DM patients had M/M genotype, a frequency comparable to that of the Italian general population [37]), despite the presence of a high prevalence of NAFLD (i.e., $71.3 \%$ had NAFLD on ultrasonography and nearly $20 \%$ had LSM $\geq 7 \mathrm{kPa}$ on FibroScan ${ }^{\circledR}$ ). Thus, it is possible to hypothesize that we did not had sufficient power to detect the adverse effects of the PNPLA3 I148M gene variant per se on both the development of NAFLD, which was already high due to T2DM itself, and its progression to NASH and advanced fibrosis, which is not a frequent event in individuals not selected for the presence of liver damage. In fact, patients with T2DM have a myriad of other non-genetic, acquired risk factors (such as obesity, insulin resistance and other metabolic syndrome features) that play important roles in the development and progression of $\operatorname{NAFLD}(1,2)$.

Collectively, the results of our study corroborate recent observations showing that regardless of established renal risk factors and the presence of NAFLD, the PNPLA3 I148M variant was strongly associated with decreasing e-GFR levels, abnormal albuminuria and higher prevalence of CKD in both adults and children (17-23). For instance, our group recently showed that carriage of the I148M variant was independently associated with decreasing eGFR levels and increasing urinary protein excretion in 142 overweight non-diabetic children with biopsy-proven NAFLD (21). Similarly, Sun et al. reported that homozygosity for the I148M variant was independently associated with an increased prevalence of CKD and abnormal albuminuria or higher levels of urinary neutrophil gelatinase-associated lipocalin (i.e., a 
marker of renal tubular injury) in 217 Chinese adults with biopsy-proven NAFLD (20).

Moreover, in a cross-sectional study of 77 post-menopausal T2DM women, Mantovani et al. reported that homozygosity for the $148 \mathrm{M}$ variant was independently associated with lower

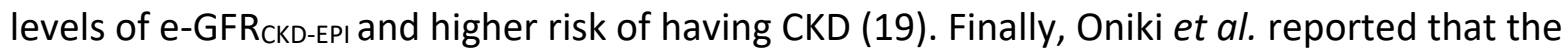
PNPLA3 I148M variant was significantly associated with a decline in kidney function over a mean follow-up of $\sim 5$ years in Japanese elderly individuals (18).

PNPLA3 is highly expressed on the lipid droplets of hepatocytes and it is involved in the development and progression of NAFLD $(6-8,38)$. Specifically, the I148M allele of the PNPLA3 gene impairs the phospholipase activity of the enzyme and promotes liver fat accumulation, by accumulating at the surface of lipid droplets, where it interacts with comparative gene identification-58 (CGI-58), which is an essential cofactor for the adipose triglyceride lipase, thereby impairing hepatic lipid remodeling (39). However, accumulating evidence indicates that PNPLA3 is highly expressed in hepatic stellate cells, i.e., the liver pericytes representing the main body storage of retinoids, which regulate blood flow in liver sinusoids in physiological conditions (40). In response to liver damage, hepatic stellate cells are activated from a quiescent to a pro-fibrotic state $(41,42)$. This process is associated with the release of retinol from intracellular lipid droplets, a process that requires PNPLA3, whereas the I148M variant impairs this process, thus resulting in a pro-inflammatory and pro-fibrotic liver phenotype (43-45). To date, while the role of the PNPLA3 I148M variant in the development and progression of NAFLD is beginning to be unraveled, the mechanisms underpinning the association between the I148M allele and the decline in e-GFR levels are poorly understood and remain largely speculative. A plausible explanation for this finding is that this association is mediated by the presence and severity of NAFLD. However, the results of our study and 
those of other recently published studies suggest that the association between the PNPLA3 I148M variant and decreased kidney function was independent of the shared renal/metabolic risk factors and the presence of hepatic steatosis and severity of liver fibrosis (18-22). Although we could not rule out that the impact of the PNPLA3 I148M variant on kidney damage is mediated by quantitative or qualitative alterations of hepatic fat content leading to the release of inflammatory mediators, it is therefore possible to hypothesize that the PNPLA3 I148M variant might exert adverse effects on the kidney $(19-21,40)$. To test this hypothesis, we have assessed PNPLA3 mRNA and protein expression levels in various human tissues and performed experiments using human cell lines representing the major renal cell populations. We included both renal podocytes that are mainly found in the glomeruli of the renal cortex and HK2 cells that are an immortalized proximal tubule epithelial cell line from normal human kidney. Across the human tissues studied, we found that the PNPLA3 mRNA expression levels were highest in the liver and in the renal cortex. In keeping with these data, among the major cellular types of the kidney, we found that podocytes were characterized by higher PNPLA3 mRNA and, in particular, protein expression levels, compared to renal tubular cells and other cell types (including hepatocytes). We hypothesize that different post-translational protein regulation may account for the different ratios between PNPLA3 mRNA and protein levels across cell types under our experimental conditions. However, it is important to note that the PNPLA3 protein expression in podocytes was comparable to that of hepatic stellate cells, which are known to express this protein at the highest level in the liver $(6-8,38)$. Interestingly, kidney damage leads to the activation of renal podocytes and pericytes, which, similarly to hepatic stellate cells, are stromal cells partially covering capillary walls that play a key role both in angiogenesis and in regulating renal medullary and cortical blood flows, thus promoting kidney fibrogenesis and glomerulosclerosis $(46,47)$. Thus, our findings are 
consistent with the hypothesis that carriage of the PNPLA3 I148M variant adversely affects kidney function, independent of the presence of significant hepatic fibrosis, possibly through a direct effect on renal podocytes. Although it is known that serum retinol concentrations are increased in patients with CKD (48), scant data are currently available on the regulation of lipid and retinol metabolism in renal podocytes $(46,47)$. However, recent data suggest that podocytes can accumulate lipid droplets, and that this process is associated with activation of the inflammasome and is involved in the pathogenesis of glomerulopathy associated with T2DM and obesity $(24,49-51)$. Consistently with decreased retinol bioavailability in pericytes carrying the PNPLA3 I148M variant (43-45), it has been reported that a highly selective agonist for retinoic acid receptor $\beta 2$ (i.e., AC261) was able to ameliorate diabetic nephropathy in an experimental mouse model (52). However, further mechanistic studies are needed to examine the role of PNPLA3 and the impact of the II48M variant on retinol and lipid metabolism in the kidney, as well as on the inflammatory and fibrogenic responses to injury in renal podocytes.

If confirmed in future studies, our observations may have important clinical implications, because they supports the assertion that PNPLA3 genotyping might be useful not only for identifying subjects with greater susceptibility to NAFLD development and progression, but also for identifying those patients with NAFLD who are at higher risk of developing CKD, thus facilitating the implementation of specific prevention programs and treatment strategies for CKD among the carriers of the PNPLA3 I148M variant.

Our study has some other important limitations that should be mentioned. First, the crosssectional design of the study limits establishing temporal or causal relationships between the PNPLA3 I148M variant and risk of CKD. However, this limitation is mitigated by the fact that 
this genetic variant is inherited and, therefore, reverse causation does not apply. Second, although we did not perform a liver biopsy, the diagnosis of NAFLD was performed by ultrasonography and vibration-controlled transient elastography, which are two imaging methods widely used in routine clinical practice (53). Third, although we did not directly measure GFR (by plasma iohexol disappearance), we used the two most widely accepted serum creatinine-based equations for estimating GFR in adults $(25,26)$. Fourth, our patient cohort comprised exclusively Caucasian individuals with non-insulin-treated T2DM without known liver diseases, attending a diabetes outpatient service. Thus, results cannot be necessarily extrapolated to the general population of patients with T2DM or other ethnic groups. Finally, we cannot definitely exclude residual confounding as a result of unmeasured risk factors.

Despite these limitations, our study has also important strengths, including the relatively large sample size, the consecutive enrollment of the study population, the completeness of the database, the adjustment for established renal risk factors, the use of vibration-controlled transient elastography for measuring liver stiffness, as well as the exclusion of patients with important comorbidities (e.g., advanced kidney disease, cirrhosis or active cancer). In addition, this is the first study that has examined the PNPLA3 mRNA and protein expression levels in human cell lines representing the major renal cell populations.

In conclusion, the results of this study show that in patients with T2DM, the PNPLA3 I148M variant was associated with decreasing e-GFR levels and higher risk of having CKD. These associations were independent of established renal risk factors, diabetes-related variables and the severity of NAFLD. Our findings also show that PNPLA3 was expressed at high levels in the 
renal cortex, mainly in podocytes. However, more research is needed to better understand the role of the PNPLA3 I148M variant in the development and progression of kidney damage and the function of the I148M mutant protein in the kidney.

\section{REFERENCES}

1. Lonardo A, Bellentani S, Argo CK, Ballestri S, Byrne CD, Caldwell SH, et al. Epidemiological modifiers of non-alcoholic fatty liver disease: focus on high-risk groups. Dig Liver Dis 2015;47:997-1006.

2. Younossi Z, Anstee QM, Marietti M, Hardy T, Henry L, Eslam M, et al. Global burden of NAFLD and NASH: trends, predictions, risk factors and prevention. Nat Rev Gastroenterol Hepatol 2018;15:11-20.

3. Webster AC, Nagler EV, Morton RL, Masson P. Chronic kidney disease. Lancet 2017;389:1238-1252.

4. Glassock RJ, Warnock DG, Delanaye P. The global burden of chronic kidney disease: estimates, variability and pitfalls. Nat Rev Nephrol 2017;13:104-114.

5. Go AS, Chertow GM, Fan D, McCulloch CE, Hsu CY. Chronic kidney disease and the risks of death, cardiovascular events, and hospitalization. N Engl J Med 2004;351:12961305.

6. Anstee QM, Seth D, Day CP. Genetic factors that affect risk of alcoholic and nonalcoholic fatty liver disease. Gastroenterology 2016;150:1728-1744.

7. Romeo S, Kozlitina J, Xing C, Pertsemlidis A, Cox D, Pennacchio LA, et al. Genetic variation in PNPLA3 confers susceptibility to nonalcoholic fatty liver disease. Nat Genet 2008;40:1461-1465. 
8. Donati B, Motta BM, Pingitore P, Meroni M, Pietrelli A, Alisi A, et al. The rs2294918 E434K variant modulates patatin-like phospholipase domain-containing 3 expression and liver damage. Hepatology 2016;63:787-798.

9. Targher G, Chonchol M, Bertolini L, Rodella S, Zenari L, Lippi G, et al. Increased risk of CKD among type 2 diabetics with nonalcoholic fatty liver disease. J Am Soc Nephrol 2008;19:1564-1570.

10. Targher G, Mantovani A, Pichiri I, Mingolla L, Cavalieri V, Mantovani W, et al. Nonalcoholic fatty liver disease is independently associated with an increased incidence of chronic kidney disease in patients with type 1 diabetes. Diabetes Care 2014;37:1729-1736.

11. Huh JH, Kim JY, Choi E, Kim JS, Chang Y, Sung KC. The fatty liver index as a predictor of incident chronic kidney disease in a 10-year prospective cohort study. PLoS One 2017;12:e0180951.

12. Sinn DH, Kang D, Jang HR, Gu S, Cho SJ, Paik SW, et al. Development of chronic kidney disease in patients with non-alcoholic fatty liver disease: a cohort study. J Hepatol 2017;67:1274-1280.

13. Jang HR, Kang D, Sinn DH, Gu S, Cho SJ, Lee JE, et al. Nonalcoholic fatty liver disease accelerates kidney function decline in patients with chronic kidney disease: a cohort study. Sci Rep 2018;8:4718.

14. Park H, Dawwas GK, Liu X, Nguyen MH. Nonalcoholic fatty liver disease increases risk of incident advanced chronic kidney disease: a propensity-matched cohort study. J Intern Med 2019;286:711-722. 
15. Musso G, Gambino R, Tabibian JH, Ekstedt M, Kechagias S, Hamaguchi M, et al. Association of non-alcoholic fatty liver disease with chronic kidney disease: a systematic review and meta-analysis. PLoS Med 2014;11:e1001680.

16. Mantovani A, Zaza G, Byrne CD, Lonardo A, Zoppini G, Bonora E, et al. Nonalcoholic fatty liver disease increases risk of incident chronic kidney disease: A systematic review and meta-analysis. Metabolism 2018;79:64-76.

17. Musso G, Cassader M, Gambino R. PNPLA3 rs738409 and TM6SF2 rs58542926 gene variants affect renal disease and function in nonalcoholic fatty liver disease. Hepatology 2015;62:658-659.

18. Oniki K, Saruwatari J, Izuka T, Kajiwara A, Morita K, Sakata M, et al. Influence of the PNPLA3 rs738409 polymorphism on non-alcoholic fatty liver disease and renal function among normal weight subjects. PLoS One 2015;10:e0132640.

19. Mantovani A, Zusi C, Sani E, Colecchia A, Lippi G, Zaza GL, et al. Association between PNPLA3 rs738409 polymorphism and decreased kidney function in postmenopausal type 2 diabetic women with or without non-alcoholic fatty liver disease. Diabetes Metab 2019;45:480-487.

20. Sun DQ, Zheng KI, Xu G, Ma HL, Zhang HY, Pan XY, et al. PNPLA3 rs738409 is associated with renal glomerular and tubular injury in NAFLD patients with persistently normal ALT levels. Liver Int 2020;40:107-119.

21. Targher G, Mantovani A, Alisi A, Mosca A, Panera N, Byrne CD, et al. Relationship Between PNPLA3 rs738409 polymorphism and decreased kidney function in children with NAFLD. Hepatology 2019;70:142-153.

22. Di Costanzo A, Pacifico L, D'Erasmo L, Polito L, Martino MD, Perla FM, et al. Nonalcoholic fatty liver disease (NAFLD), but not its susceptibility gene variants, 
influences the decrease of kidney function in overweight/obese children. Int J Mol Sci 2019;20(18).pii: E4444.

23. Marzuillo P, Di Sessa A, Guarino S, Capalbo D, Umano GR, Pedullà M, et al. Nonalcoholic fatty liver disease and eGFR levels could be linked by the PNPLA3 I148M polymorphism in children with obesity. Pediatr Obes 2019:e12539.

24. Zhao J, Rui HL, Yang M, Sun L, Dong HR, Cheng H. CD36-mediated lipid accumulation and activation of NLRP3 inflammasome lead to podocyte injury in obesity-related glomerulopathy. Mediators Inflamm 2019;2019:3172647.

25. Mantovani A, Sani E, Fassio A, Colecchia A, Viapiana O, Gatti D, et al. Association between non-alcoholic fatty liver disease and bone turnover biomarkers in postmenopausal women with type 2 diabetes. Diabetes Metab 2019;45:347-355.

26. Levey AS, Stevens LA, Schmid CH, Zhang YL, Castro AF 3rd, Feldman HI, et al; CKD-EPI (Chronic Kidney Disease Epidemiology Collaboration). A new equation to estimate glomerular filtration rate. Ann Intern Med 2009;150:604-612.

27. Levey AS, Bosch JP, Lewis JB, Greene T, Rogers N, Roth D. A more accurate method to estimate glomerular filtration rate from serum creatinine: a new prediction equation. Modification of Diet in Renal Disease Study Group. Ann Intern Med 1999;130:461-470.

28. American Diabetes Association. Microvascular complications and foot care: standards of medical care in diabetes-2019. Diabetes Care 2019;42(suppl 1):S124-S138.

29. Colecchia A, Montrone L, Scaioli E, Bacchi-Reggiani ML, Colli A, Casazza G, et al. Measurement of spleen stiffness to evaluate portal hypertension and the presence of esophageal varices in patients with HCV-related cirrhosis. Gastroenterology 2012;143:646-654. 
30. Castera L, Friedrich-Rust M, Loomba R. Noninvasive assessment of liver disease in patients with nonalcoholic fatty liver disease. Gastroenterology 2019;156:1264-1281.

31. Myers RP, Pomier-Layrargues G, Kirsch R, Pollett A, Duarte-Rojo A, Wong D, et al. Feasibility and diagnostic performance of the FibroScan XL probe for liver stiffness measurement in overweight and obese patients. Hepatology 2012;55:199-208.

32. Puigvehí M, Broquetas T, Coll S, Garcia-Retortillo M, Cañete N, Fernández R, et al. Impact of anthropometric features on the applicability and accuracy of FibroScan ${ }^{\circledR}(\mathrm{M}$ and XL) in overweight/obese patients. J Gastroenterol Hepatol. 2017;32:1746-1753.

33. Carvalhana S, Leitão J, Alves AC, Bourbon M, Cortez-Pinto H. How good is controlled attenuation parameter and fatty liver index for assessing liver steatosis in general population: correlation with ultrasound. Liver Int 2014;34:e111-117.

34. McCall MN, Uppal K, Jaffee HA, Zilliox MJ, Irizarry RA. The gene expression barcode: leveraging public data repositories to begin cataloging the human and murine transcriptomes. Nucleic Acids Res 2011; 9 (database issue):D1011-5.

35. Saleem MA, O'Hare MJ, Reiser J, Coward RJ, Inward CD, Farren T, et al. A conditionally immortalized human podocyte cell line demonstrating nephrin and podocin expression. J Am Soc Nephrol 2002;13:630-638.

36. Pingitore $P$, Pirazzi $C$, Mancina RM, Motta BM, Indiveri C, Pujia A, et al. Recombinant PNPLA3 protein shows triglyceride hydrolase activity and its I148M mutation results in loss of function. Biochim Biophys Acta 2014;1841:574-580.

37. Valenti L, Rametta R, Ruscica M, Dongiovanni P, Steffani L, Motta BM, et al. The I148M PNPLA3 polymorphism influences serum adiponectin in patients with fatty liver and healthy controls. BMC Gastroenterol. 2012;12:111. 
38. Eslam M, Valenti L, Romeo S. Genetics and epigenetics of NAFLD and NASH: clinical impact. J Hepatol 2018;68:268-279.

39. Wang Y, Kory N, BasuRay S, Cohen JC, Hobbs HH. PNPLA3, CGI-58, and inhibition of hepatic triglyceride hydrolysis in mice. Hepatology 2019;69:427-2441.

40. Romeo S, Dongiovanni P, Petta S, Pihalajamaki J, Valenti L. Reply: To PMID 25251399. Hepatology 2015;62:660.

41. Friedman SL. Hepatic stellate cells: protean, multifunctional, and enigmatic cells of the liver. Physiol Rev 2008;88:125-172.

42. Poisson J, Lemoinne S, Boulanger C, Durand F, Moreau R, Valla D, et al. Liver sinusoidal endothelial cells: physiology and role in liver diseases. J Hepatol 2017;66:212-227.

43. Pingitore $\mathrm{P}$, Dongiovanni $\mathrm{P}$, Motta BM, Meroni M, Lepore SM, Mancina RM, et al. PNPLA3 overexpression results in reduction of proteins predisposing to fibrosis. Hum Mol Genet 2016;25:5212-5222.

44. Mondul A, Mancina RM, Merlo A, Dongiovanni P, Rametta R, Montalcini T, et al. PNPLA3 I148M variant influences circulating retinol in adults with nonalcoholic fatty liver disease or obesity. J Nutr 2015;145:1687-1691.

45. Bruschi FV, Claudel T, Tardelli M, Caligiuri A, Stulnig TM, Marra F, et al. The PNPLA3 I148M variant modulates the fibrogenic phenotype of human hepatic stellate cells. Hepatology 2017;65:1875-1890.

46. Kramann R, Humphreys BD. Kidney pericytes: roles in regeneration and fibrosis. Semin Nephrol 2014;4:374-383.

47. Shaw I, Rider S, Mullins J, Hughes J, Peault B. Pericytes in the renal vasculature: roles in health and disease. Nat Rev Nephrol 2018;14:521-534. 
48. Hamamura $\mathrm{K}$, Matsunaga $\mathrm{N}$, Ikeda E, Kondo $\mathrm{H}$, Ikeyama $\mathrm{H}$, Tokushige $\mathrm{K}$, et al.

Alterations of hepatic metabolism in chronic kidney disease via d-box-binding protein aggravate the renal dysfunction. J Biol Chem 2016;291:4913-4927.

49. Mallela SK, Patel DM, Ducasa GM, Merscher S, Fornoni A, Al-Ali H. Detection and quantification of lipid droplets in differentiated human podocytes. Methods Mol Biol 2019;1996:199-206.

50. Zhang Y, Ma KL, Liu J, Wu Y, Hu ZB, Liu L, et al. Inflammatory stress exacerbates lipid accumulation and podocyte injuries in diabetic nephropathy. Acta Diabetol $2015 ; 52: 1045-1056$.

51. McPherson KC, Taylor L, Johnson AC, Didion SP, Geurts AM, Garrett MR, et al. Early development of podocyte injury independently of hyperglycemia and elevations in arterial pressure in nondiabetic obese Dahl SS leptin receptor mutant rats. Am J Physiol Renal Physiol 2016;311:F793-F804.

52. Trasino SE, Tang XH, Shevchuk MM, Choi ME, Gudas LJ. Amelioration of diabetic nephropathy using a retinoic acid receptor $\beta 2$ agonist. J Pharmacol Exp Ther 2018;367:82-94.

53. Byrne CD, Patel J, Scorletti E, Targher G. Tests for diagnosing and monitoring nonalcoholic fatty liver disease in adults. BMJ 2018;362:k2734. 
Table 1. Main clinical and biochemical characteristics of patients with non-insulin-treated T2DM, stratified by PNPLA3 I148M variant.

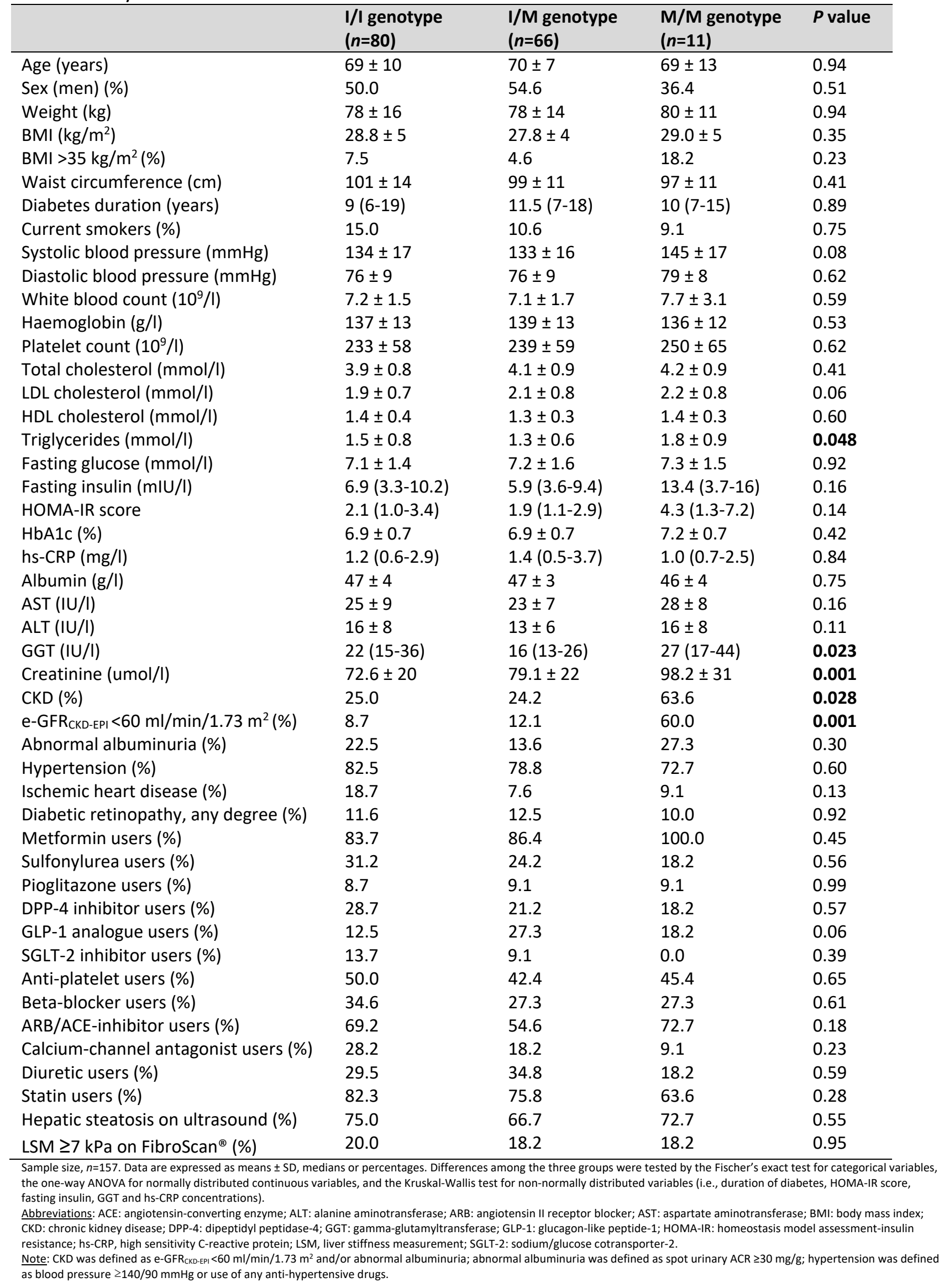


Table 2. Linear Regression Analysis - Effect of PNPLA3 I148M variant on e-GFR $\mathrm{RKD}_{\text {-EPI }}$ levels in patients with T2DM.

\section{$B$ coefficient(s) $\quad 95 \% \mathrm{Cl}(\mathrm{s}) \quad$ Standardized $\quad P$ value} B coefficient(s)

\begin{tabular}{|c|c|c|c|c|}
\hline Unadjusted linear & & & & \\
\hline e-GFR ${ }_{\text {CKD-EPI }}$ for $1 \mathrm{~m}$ & & & & \\
\hline PNPLA3 rs738409 & & & & \\
\hline I/I genotype & Ref. & Ref. & & - \\
\hline I/M genotype & -5.61 & -10.8 to -0.36 & -0.16 & 0.026 \\
\hline M/M genotype & -22.9 & -33.5 to -12.3 & -0.33 & $<0.0001$ \\
\hline e-GFR CKD-EPI $_{\text {for } 1 \mathrm{~m}}$ & & & & \\
\hline PNPLA3 rs738409 & -8.49 & -12.6 to -4.34 & -0.31 & $<0.0001$ \\
\hline Adjusted linear reg & & & & \\
\hline e-GFR CKD-EPI $_{\text {for } 1 \mathrm{~m}}$ & & & & \\
\hline PNPLA3 rs738409 & & & & \\
\hline I/I genotype & Ref. & Ref. & & - \\
\hline I/M genotype & -5.77 & -10.8 to -0.72 & -0.17 & 0.025 \\
\hline M/M genotype & -23.2 & -33.2 to -13.2 & -0.34 & $<0.0001$ \\
\hline e-GFR CKD-EPI $_{\text {for } 1 \text { r }}$ & & & & \\
\hline PNPLA3 rs738409 & -8.71 & -12.7 to -4.70 & -0.32 & $<0.0001$ \\
\hline
\end{tabular}

Sample size, $n=157$. Data are expressed as beta coefficients and $95 \%$ confidence intervals $(\mathrm{Cl})$ as tested by linear regression analysis. The continuous values of e-GFR (for every $1 \mathrm{ml} / \mathrm{min} / 1.73 \mathrm{~m}^{2}$ decrement) were included as the dependent variable in all linear regression models. The impact of PNPLA3 rs738409, encoding for the I148M protein variant, on levels of e-GFR was assessed using an additive genetic model. Ref. = reference category.

*This multivariable linear regression model was adjusted, along with PNPLA3 rs738409 genotypes, for the following covariates: duration of diabetes, $\mathrm{HbA1c}$, hypertension (i.e., blood pressure $\geq 140 / 90 \mathrm{mmHg}$ or use of any anti-hypertensive drugs), presence of LSM $\geq 7$ kPa on FibroScan ${ }^{\circledR}$ and abnormal albuminuria (i.e., spot urinary $A C R \geq 30 \mathrm{mg} / \mathrm{g}$ creatinine). 
Table 3. Logistic Regression Analysis - Effect of PNPLA3 I148M variant on risk of prevalent CKD in patients with T2DM.

\begin{tabular}{|c|c|c|c|}
\hline & Odds Ratio(s) & $95 \% \mathrm{Cl}(\mathrm{s})$ & $P$ value \\
\hline \multicolumn{4}{|c|}{ Unadjusted logistic regression model } \\
\hline \multicolumn{4}{|l|}{ CKD (yes vs. no) } \\
\hline \multicolumn{4}{|l|}{ PNPLA3 rs738409 } \\
\hline I/I genotype & Ref. & Ref. & - \\
\hline I/M genotype & 0.96 & $0.45-2.04$ & 0.92 \\
\hline M/M genotype & 5.27 & $1.39-19.8$ & 0.014 \\
\hline \multicolumn{4}{|c|}{ Adjusted logistic regression model * } \\
\hline \multicolumn{4}{|l|}{ CKD (yes vs. no) } \\
\hline \multicolumn{4}{|l|}{ PNPLA3 rs738409 } \\
\hline I/I genotype & Ref. & Ref. & - \\
\hline I/M genotype & 0.99 & $0.45-2.14$ & 0.96 \\
\hline M/M genotype & 6.65 & $1.65-26.8$ & 0.008 \\
\hline \multicolumn{4}{|c|}{$\begin{array}{l}\text { Sample size, } n=157 \text {. Data are expressed as odds ratio(s) and } 95 \% \text { confidence intervals }(\mathrm{Cl}) \text { as tested by logistic regression analysis. The } \\
\text { presence of CKD was included as the dependent variable in all logistic regression models. The impact of PNPLA3 rs } 738409 \text {, encoding for the } \\
\text { I148M protein variant, on risk of CKD was assessed using an additive genetic model. Ref. = reference category. }\end{array}$} \\
\hline
\end{tabular}




\section{FIGURE LEGENDS}

Fig. 1. Box plot of e-GFR T2DM, stratified by PNPLA3 I148M variant. $P$-values for the trend by the Kruskal-Wallis test. The central rectangle spans the first quartile to the third quartile (the IQR). The segment inside the rectangle shows the median, and the whiskers above and below the box indicate the locations of $1.5 \times$ IQR values.

Fig. 2. PNPLA3 rs738409 expression in human organs and tissues. Panel A: Z-score values of PNPLA3 expression in tissues. The dash line indicates the threshold above which a gene is considered expressed. A Z-score $>5$ suggests that an annotated gene is expressed in a specific tissue. Other tissues refer to the mean of Z-scores of tissues that do not include kidney and the liver. Panel B: Genotype and PNPLA3 mRNA expression level in HepG2 hepatocytes, LX2 (hepatic stellate cells) and conditionally immortalized human renal podocytes, HK2 (renal tubular cells) and Human Umbilical Vein Endothelial cells (HUVEC). Panel C: PNPLA3 protein expression in HepG2 hepatocytes, LX2 (hepatic stellate cells) and conditionally immortalized human renal podocytes, HK2 (tubular cells) and Human Umbilical Vein Endothelial cells (HUVEC). Panel D: PNPLA3 protein quantification in HepG2 hepatocytes, LX2 (hepatic stellate cells) and conditionally immortalized human renal podocytes, HK2 (tubular cells) and Human Umbilical Vein Endothelial cells (HUVEC).

NB: in all panels above: $\left({ }^{*}\right)$ represents the statistical significance of values normalized to HepG2 hepatocyte control, whereas $\left({ }^{\bullet}\right)$ represents the statistical significance of values normalized to podocyte control. 